\title{
Synthesis of novel phases in Si nanowires using diamond anvil cells at high pressures and temperatures
}

Larissa Q. Huston ${ }^{1, a}$, Alois Lugstein², Guoyin Shen ${ }^{3}$, David A. Cullen4, Bianca Haberl ${ }^{5}$, Jim S. Williams ${ }^{1}$ and Jodie E. Bradby ${ }^{1}$

${ }^{1}$ Department of Electronic Materials Engineering, Research School of Physics, The Australian National University, Acton, Australian Capital Territory, Australia

${ }^{2}$ Institute for Solid State Electronics, Vienna University of Technology, Floragasse 7, 1040 Vienna, Austria

${ }^{3}$ High Pressure Collaborative Access Team, X-ray Science Division, Argonne National Laboratory, Argonne, IL 60439, USA

${ }^{4}$ Center for Nanophase Materials Sciences, Physical Sciences Directorate, Oak Ridge National Laboratory, Oak Ridge, TN 37831, USA

${ }^{5}$ Neutron Scattering Division, Neutron Sciences Directorate, Oak Ridge National Laboratory, Oak Ridge, TN 37831, USA

aPresent address: Shock and Detonations Physics (M-9), Los Alamos National Laboratory, Los Alamos, New Mexico 87545, United States United States, larissa@lanl.gov

\section{Supporting information}




\section{Experimental Method}

\section{Nanowire growth}

The Si nanowires studied here were seeded with $\mathrm{Au}$ and grown in the $<111>$ direction using the vapour-liquid-solid method to a diameter $80-150 \mathrm{~nm}$ and length of $5 \mu \mathrm{m} .{ }^{1}$ The conditions used for growth can be found in ref. ${ }^{2}$ After growth, the Au was removed by etching the nanowires in hydrofluoric acid, to first remove the native oxide layer and then in aqua Regia solution.

\section{Loading of Nanowires in a diamond anvil cell}

Nanowires were removed from their substrate using a stainless steel razorblade and transferred into a symmetric diamond anvil cell (DAC) with $400 \mu \mathrm{m}$ cullet size. A stainless steel gasket was preindented to $45-60 \mu \mathrm{m}$ in thickness and then a $200 \mu \mathrm{m}$ diameter hole was laser drilled using the system at HPCAT. ${ }^{3}$ A small ruby chip was placed into the cell so that pressure could be monitored using ruby fluorescence. The DAC was then gas loaded with Ne which was used as a quasihydrostatic pressure medium and to keep the nanowires separate.

\section{Details of ex-situ measurements}

The pressure of the cell was remotely controlled using a double membrane system. ${ }^{4}$ Before increasing pressure, the DAC was heated to $25,70,105,135$ and $165^{\circ} \mathrm{C}$ using a resistive heating block and variable resister. Temperature was measured using a type $\mathrm{K}$ thermocouple which was connected on the outside of the resistive heating block. The temperature was allowed at least 1 hour to equilibrate before increasing the pressure to 20-22 GPa to ensure that the nanowires had fully phase transformed to sh-Si, in accordance to a previous study. ${ }^{2}$ This was confirmed by Raman spectroscopy, which showed no evidence for any dc-Si peaks at this pressure. Pressure was then decreased to $\sim 9-10 \mathrm{GPa}$, where the sample had transformed to $\beta$ $\mathrm{Sn}-\mathrm{Si}$, and unloaded to 2-3 GPa at a rate of $\sim 2 \mathrm{GPa} / \mathrm{hr}$. The decompression rate was monitored by measuring ruby fluorescence every $10 \mathrm{sec}$. It should be noted that it was difficult to keep the unloading rate uniform due to the gasket not responding smoothly during decompression, causing occasional drops in pressure of $\sim 0.2-0.7 \mathrm{GPa}$ due to the leaking of the pressure medium. Care was taken to minimize this effect and ensure that the unloading rate was as uniform as possible. Once the sample reached $\sim 2-3 \mathrm{GPa}$, a pressure where all the $\beta$-Sn-Si should have transformed to other phases, the DAC was cooled in air and the remaining pressure was released. The sample was then removed from the DAC (but remained in the gasket). Two $<100>$ oriented single crystal bulk-Si samples of $15 \pm 2 \mu \mathrm{m}$ thickness were also compressed under similar conditions at 105 and $165^{\circ} \mathrm{C}$.

To determine the final crystal structure of each of the samples, powder x-ray diffraction was used. This was performed at the 16-ID-B beamline at the Advanced Photon Source at Argonne National Laboratory. The energy of the $\mathrm{x}$-rays was $31 \mathrm{keV}$ for the nanowires and $25 \mathrm{keV}$ for bulk-Si. The spot size was $\sim 4 \times 6 \mu \mathrm{m}$ FHWM and a Pilatus $1 \mathrm{M}-\mathrm{F}$ detector used. The data were collected for 120-180 sec for the nanowires and $30 \mathrm{sec}$ for the bulk samples. A measurement of the background air scattering through an empty gasket was subtracted from each measurement before integrating the pattern using Dioptas. ${ }^{5}$ 


\section{Details of in-situ measurements}

A similar setup to the ex-situ measurements was used for an in-situ experiment. In addition to the SiNWs, ruby and Ne pressure medium; a small chip of Pt was loaded into a DAC. The same resistive heating block and double membrane system were used. The DAC was heated to $\sim 105^{\circ} \mathrm{C}$ and was given over an hour to stabilize in temperature. The diamond in the downstream direction was mounted on a cubic boron nitride seat to provide an X-ray transparent window for the higher angle diffracted $\mathrm{x}$-rays to pass through.

The pressure was increased until no dc-Si was observed in the x-ray powder diffraction pattern $\sim 19 \mathrm{GPa}$. The pressure was then decreased slowly at a rate of $\sim 30 \mathrm{GPa} / \mathrm{hr}$ and the phase present inside the cell was recording using $10 \mathrm{sec}$ exposures until all the $\beta$-Sn-Si phase had appeared and then disappeared. Pressure was determined by calibration using the equation of state based on the (111) reflection of Pt in ref. ${ }^{6}$

\section{Transmission Electron Microscopy}

After pressurization, the SiNWs were then transferred into a small container and then ultrasonicated in isopropanol. Afterwards, they were then transferred onto a copper lacey carbon grid using a micropipette. The nanowires were then examined using a Hitachi HF3300 transmission electron microscope operating at $300 \mathrm{kV}$ or a Philips CM300 (in the case of some of the supplementary images). Dark field images were produced by inserting an objective aperture over the reflection of interest; the size of the aperture used is indicated by the red circles on the SAED patterns associated with the additional TEM micrographs shown in Supplementary Fig. 2.

\section{Additional TEM Images}

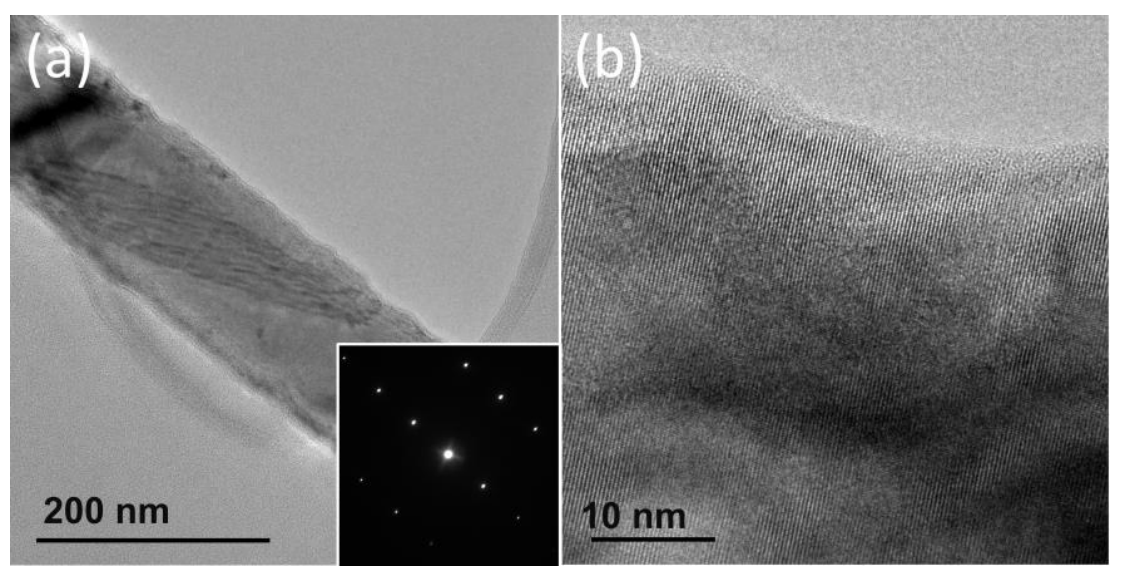

Supplementary Figure 1: Representative transmission electron microscope images of the SiNWs before compression (a) Bright field image with inset selected area diffraction pattern, (b) higher magnification image. 


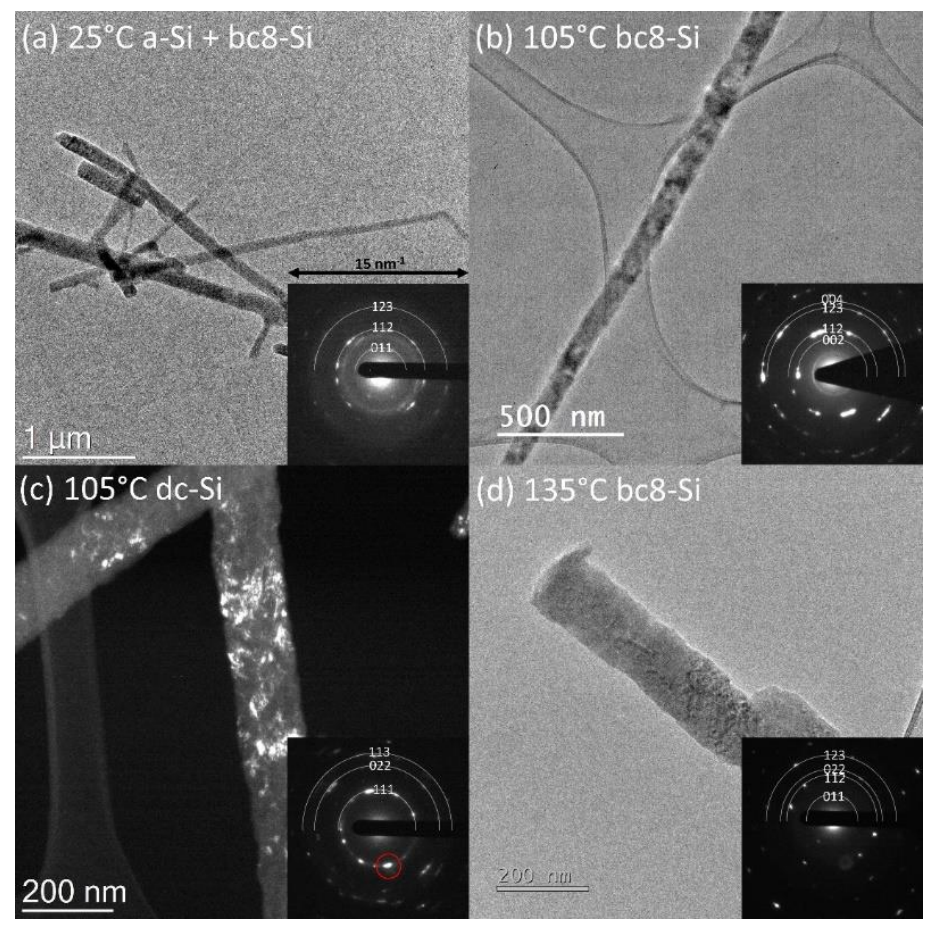

Supplementary Figure 2 Additional TEM micrographs of SiNWs after compression and decompression at (a) at $25^{\circ} \mathrm{C}$, containing bc-8-Si and a-Si; (b) at $105^{\circ} \mathrm{C}$, showing bc8-Si; (c) at $105^{\circ} \mathrm{C}$, containing dc-Si; and (d) at $135^{\circ} \mathrm{C}$, showing bc8-Si. All inset SAED patterns are 15 $\mathrm{nm}^{-1}$.

\section{References}

(1) Wagner, R. S.; Ellis, W. C. Vapor Liquid Solid Mecahism of Single Crystal Growth. Appl. Phys. Lett. 1964, 4 (5), 89-90.

(2) Huston, L. Q.; Lugstein, A.; Bradby, J. E.; Williams, J. S. The High Pressure Phase Transformation Behaviour of Silicon Nanowires. App. Phys. Lett 2018, 113, 123103.

(3) Hrubiak, R.; Sinogeikin, S.; Rod, E.; Shen, G. The Laser Micro-Machining System for Diamond Anvil Cell Experiments and General Precision Machining Applications at the High Pressure Collaborative Access Team. Rev. Sci. Instrum. 2015, 86 (7).

(4) Sinogeikin, S. V; Smith, J. S.; Rod, E.; Lin, C.; Kenney-Benson, C.; Shen, G. Online Remote Control Systems for Static and Dynamic Compression and Decompression Using Diamond Anvil Cells. Rev. Sci. Instrum. 2015, 86 (7), 72209.

(5) Prescher, C.; Prakapenka, V. B. DIOPTAS: A Program for Reduction of TwoDimensional X-Ray Diffraction Data and Data Exploration. High Press. Res. 2015, 35 (3), 223-230.

(6) Fei, Y.; Ricolleau, A.; Frank, M.; Mibe, K.; Shen, G.; Prakapenka, V. Fei +2006 Pressure Scale PNAS.Pdf. 2007. 